\title{
MORTALIDADE POR ACIDENTES DE TRÂNSITO TERRESTRE: ANÁLISE COMPARATIVA
}

Marília Angelina Ferreira Papa ${ }^{1}$, Danielle Wisniewski ${ }^{1}$, Kelly Cristina Inoue ${ }^{1}$, Carlos Alexandre Molena-Fernandes ${ }^{2}$,
Yolanda Dora Martinez Évora ${ }^{3}$ Laura Misue Matsuda ${ }^{4}$

RESUMO: Estudo epidemiológico, descritivo, comparativo, cujo objetivo consistiu em comparar a mortalidade por Acidentes de Transporte Terrestre nas coortes: 2006/2007 e 2009/2010, em Maringá-Paraná, Brasil. Realizou-se estatística inferencial (Teste Qui-Quadrado) no programa Statistica 8.0 ${ }^{\circledR}$. Constatou-se que os Acidentes de Transporte Terrestre são os principais responsáveis pela mortalidade por causas externas; o coeficiente de mortalidade local foi de $34,98 \%$, superior ao nacional, principalmente entre pedestres, motociclistas e homens entre 20 a 39 anos. Na comparação das diferenças do coeficiente de mortalidade das coortes, houve um aumento de $26,4 \%$. Conclui-se que no município houve aumento do coeficiente de mortalidade por Acidentes de Transporte Terrestre e, sugerem-se maiores investimentos em programas de educação no trânsito, fiscalização e estudos que avaliem impacto da medida restritiva do uso de álcool e direção no trânsito. DESCRITORES: Acidentes de trânsito; Epidemiologia; Mortalidade.

\section{MORTALIDAD POR ACCIDENTES DE TRÁNSITO TERRESTRE: ANÁLISIS COMPARATIVO}

RESUMEN: Estudio epidemiológico, descriptivo, comparativo, cuyo objetivo fue comparar la mortalidad por Accidentes de Transporte Terrestre en la muestra: 2006/2007 y 2009/2010, en Maringá - Paraná, Brasil. Fue realizada estadística inferencial en el programa Statistica 8.0®. Se concluyó que los Accidentes de Transporte Terrestre son los principales responsables por la mortalidad de causas externas; el coeficiente de mortalidad local fue de $34,98 \%$, superior al nacional, principalmente entre pedestres, motociclistas y hombres entre 20 y 39 años. En la comparación de las diferencias del coeficiente de mortalidad de las muestras, hubo una elevación de $26,4 \%$. Se concluye que en el municipio hubo elevación del coeficiente de mortalidad por Accidentes de Transporte Terrestre y, la sugerencia es que se hagan más inversiones en programas de educación en el tránsito, fiscalización y estudios que evalúen el impacto de la medida restrictiva del uso de alcohol y conducción en el tránsito. DESCRIPTORES: Accidentes de tránsito; Epidemiología; Mortalidad.

\section{MORTALITY FROM LAND TRANSPORT ACCIDENTS: A COMPARATIVE ANALYSIS}

\begin{abstract}
This is an epidemiological, descriptive, comparative study whose objective was to compare the mortality from Land Transport Accidents in the cohorts: 2006/2007 and 2009/2010, in Maringá in the Brazilian State of Paraná. Inferential statistics were undertaken (Chi-squred test) using the Statistica 8.0® software. It was observed that Land Traffic Accidents are the main causes of mortality from external causes - the local coefficient of mortality was $34.98 \%$, above the national rate, principally among pedestrians, motorcyclists and men between 20 and 39 years old. And that in the comparison of the differences of the coefficient of mortality between the cohorts, there was an increase of $26.4 \%$. It is concluded that there was, in the city, an increase of the coefficient of mortality from Land Transport Accidents. Thus, further investment is suggested in traffic education programs; inspections; and in studies evaluating the impact of the measure restricting the use of alcohol and driving in the traffic. DESCRIPTORS: Traffic accidents; Epidemiology; Mortality.
\end{abstract}

\footnotetext{
${ }^{1}$ Enfermeira. Mestre em Enfermagem. Universidade Estadual de Maringá. Paranavaí-PR-Brasil

${ }^{2}$ Profissional de Educação Física. Doutor em Ciências Farmacêuticas. Professor da Universidade Estadual do Paraná. Paranavaí-PR-Brasil ${ }^{3}$ Enfermeira. Doutora em Enfermagem. Professora da Universidade de São Paulo. Ribeirão Preto-SP-Brasil

${ }^{4}$ Enfermeira. Doutora em Enfermagem. Professora da Universidade Estadual de Maringá. Maringá-PR-Brasil
} 


\section{INTRODUÇÃO}

No cenário brasileiro, devido ao elevado impacto na morbimortalidade, principalmente na população jovem do sexo masculino, a mortalidade por causas externas - consideradas como evitáveis ou reduzíveis - representam um sério problema de saúde pública ${ }^{(1)}$. Essas causas são definidas como

aquelas preveníveis, total ou parcialmente, por ações efetivas dos serviços de saúde que estejam acessíveis em um determinado local e época ${ }^{(2: 174)}$.

Em 2010, as causas externas constituíram o terceiro grupo de causas de morte no Brasil, passando a ocupar a primeira posição quando a análise se restringiu ao grupo de pessoas de um a 39 anos de idade ${ }^{(3)}$. Esse dado é preocupante, pois além da perda de anos potenciais de vida, as causas externas podem resultar em sequelas, com consequente aumento da demanda pelos serviços de saúde nos seus diversos níveis de complexidade assistencial, e aumento do ônus financeiro que, em 2011, se aproximou de um bilhão de reais ${ }^{(4)}$.

Dentre os óbitos por causas externas, os Acidentes de Transporte Terrestres (ATT), codificados de V01 a V89 pela CID-10 ${ }^{(5)}$, representaram, em 2010, o segundo lugar na mortalidade no Brasil, com aproximadamente 42.844 mortes $^{(4)}$.

O Brasil tem se preocupado com a prevenção dos acidentes de trânsito e em 2010, elaborou uma proposta para a construção de um Plano Nacional de Redução de Acidentes e Segurança Viária, pautado em cinco pilares: Fiscalização; Educação; Saúde; Infraestrutura e Segurança Veicular ${ }^{(6)}$.

Ressalta-se que, no contexto brasileiro, a precária conservação das estradas; longo tempo de uso da frota e sua manutenção inadequada; introdução crescente da motocicleta como instrumento de trabalho, dentre outras, têm contribuído para a ocorrência de acidentes ${ }^{(1)}$. Ademais, o álcool se destaca na determinação dos ATT, o que tem motivado diversos países, inclusive o Brasil, ao estabelecimento de medidas legais e campanhas na mídia para a redução dos efeitos da associação entre bebida alcoólica e direção, com foco direcionado, especialmente, à conscientização dos motoristas jovens ${ }^{(7)}$.

No Brasil, na perspectiva referida, em 19 de junho de 2008, foi regulamentada a Lei n. $11.705^{(8)}$, conhecida como Lei Seca, a qual alterou o Código de Trânsito Brasileiro por meio da introdução de dispositivos legais que inibem o consumo de bebida alcoólica pelo condutor de veículo automotor. Apesar da implantação da Lei mencionada, os ATT, no ano de 2010, resultaram em $12,6 \%$ de mortes precoces evitáveis no país e, ao inserir as mortes por ATT no grupo de causas externas, o número se elevou para $30 \%$, no mesmo período ${ }^{(4)}$. Em Maringá-Paraná, cujo município possuía 357.077 mil habitantes no ano de 2010, os ATT ocuparam a primeira posição de morte relacionada às causas externas, totalizando 162 óbitos. Verificou-se, ainda, que essa causa de morte aumentou em 1\% no período de 2008 a 2010 no referido município ${ }^{(4)}$.

Diante da problemática apresentada, a análise da mortalidade por ATT é importante; os dados possibilitam a tomada de decisões mais efetivas, nos diferentes segmentos da sociedade, à prevenção e redução da sua ocorrência e prejuízos. Nesse contexto, este estudo se ancora na seguinte questão: Como se apresentou a mortalidade por ATT nas coortes de 2006/2007 e de 2009/2010 no Município de Maringá? $\mathrm{E}$, para responder a essa questão, o presente estudo tem por objetivo comparar a mortalidade por ATT, ocorrida nas coortes de 2006/2007 e de 2009/2010, em Maringá - Paraná, Brasil.

\section{MÉTODO}

Estudo epidemiológico, descritivo, comparativo, baseado em dados secundários acerca da mortalidade por acidentes de transporte terrestre ocorridos no $\mathrm{Mu}$ nicípio de Maringá - Paraná, nas coortes de 2006/2007 e 2009/2010. Elegeu-se esse período em virtude da implantação da Lei n. 11.705, de 19 de junho de $2008^{(7)}$, a qual estabelece medida restritiva de consumo de bebida alcoólica pelo condutor de veículo automotor.

Os dados de mortalidade foram obtidos do Sistema de Informações sobre Mortalidade do Ministério da Saúde, através do Departamento de Informática do Sistema Único de Saúde (DATASUS $)^{(4)}$. Deste modo, foram selecionados os óbitos, no período de 2006 a 2010 na referida cidade, cuja causa básica tenha sido ATT por ocorrência, identificados pelo Capítulo XX, códigos V01 a V89 da CID-10 ${ }^{(4)}$, disponibilizados no sítio do DATASUS.

Procedeu-se uma análise de completude dos bancos de dados dos estados brasileiros do Paraná e de Maringá, em relação ao número de óbitos totais, por causas externas e por ATT. Nesse processo, foram excluídos os registros onde não constavam informações referentes ao sexo e idade da pessoa que fora a 
óbito, procedendo-se ao agrupamento desta última variável, em quatro categorias de faixa etária ( 0 a 19 anos; 20 a 39 anos; 40 a 59 anos e 60 anos e mais).

Os dados populacionais também foram obtidos na página eletrônica do DATASUS. Para o primeiro período analisado foi utilizada a média das populações dos anos de 2006 e 2007 e no segundo período a média dos anos de 2009 e 2010. As variações da mortalidade por ATT no período de 2006/2007 e 2009/2010 foram verificadas por meio da diferença dos coeficientes de mortalidade, com a finalidade de identificar a variação percentual nas taxas de mortalidade nos períodos investigados. Para o cálculo do coeficiente de mortalidade utilizou-se fórmula já padronizada, que consiste na divisão do total de óbitos por ATT pela população de residentes na mesma área e no mesmo período, multiplicando-se o resultado por 100.000 habitantes.

As análises estatísticas foram realizadas por meio do software Statistica $8.0^{\circledR}$, no qual se utilizou o teste estatístico qui-quadrado $(\chi 2)$ para medida de associação entre as variáveis, calculando-se a razão de chance, ou seja, o odds ratio (OR). O nível de significância adotado para as análises foi de 5\%, com intervalo de confiança (IC) de 95\%. A saber, a escolha pelo referido teste se deve ao desfecho dicotômico (Óbito e Não óbito).

Os dados utilizados nesta pesquisa constam em bancos dedados públicos. Desta forma, nãofoinecessário submeter o protocolo desta análise ao Comitê de Ética em Pesquisa.

\section{RESULTADOS}

No ano de 2006 foram registrados 60.560 óbitos no Paraná. Destes, 2.969 era por ATT, o que correspondeu a 4,9\% dos óbitos. Em Maringá, neste mesmo período, foram registrados 95 óbitos também por ATT, correspondendo a 3,19\% dos óbitos por acidentes do Estado e a 3,71\% dos óbitos no município. Ainda, neste mesmo ano, os ATT ocuparam a primeira posição de morte relacionada às causas externas em Maringá.

No tocante ao ano de 2010 foram registrados 67.284 óbitos no Paraná. Destes, 3342 foram devido aos ATT, o que correspondeu a 4,96\% dos óbitos. Em Maringá, neste mesmo período, foram registrados 162 óbitos por ATT, correspondendo a 4,84\% dos óbitos por acidentes do Estado e a 5,41\% dos óbitos no próprio município, o que justificou a permanência dos ATT como a principal causa de morte nesse local ${ }^{(4)}$. A distribuição de óbitos por ATT no período de 2006 a 2010 no município, segundo as categorias da CID-10 (V01-V89), está demonstrada na Tabela 1.

Na Figura 1, consta o coeficiente de mortalidade por ATT de 2006 a 2010, de acordo com o sexo.

Tabela 1- Distribuição dos óbitos por Acidentes de Transporte Terrestre, segundo categorias da CID-10 (V01-V89) e ano de ocorrência. Maringá-PRBrasil, 2006-2010

\begin{tabular}{|c|c|c|c|c|c|c|c|c|c|c|}
\hline ANO & & & & & & & & & & \\
\hline CID-10 & $\mathbf{n}$ & $\%$ & $\mathbf{n}$ & $\%$ & $\mathbf{n}$ & $\%$ & $\mathbf{n}$ & $\%$ & $\mathbf{n}$ & $\%$ \\
\hline $\begin{array}{l}\text { Pedestre traumatizado } \\
\text { em AT* }\end{array}$ & 38 & 40 & 37 & 35,24 & 44 & 37,29 & 35 & 32,71 & 50 & 30,87 \\
\hline $\begin{array}{l}\text { Motociclista traumati- } \\
\text { zado em AT* }\end{array}$ & 26 & 27,36 & 34 & 32,38 & 36 & 30,50 & 28 & 26,16 & 44 & 27,16 \\
\hline $\begin{array}{l}\text { Ocupante automóvel } \\
\text { traumatizado em AT* }\end{array}$ & 13 & 13,69 & 26 & 24,77 & 26 & 22,04 & 29 & 27,10 & 54 & 33,34 \\
\hline $\begin{array}{l}\text { Outros acidentes de } \\
\text { transporte terrestre }\end{array}$ & 13 & 13,69 & 1 & 0,95 & 4 & 3,39 & 8 & 7,48 & 9 & 5,55 \\
\hline $\begin{array}{l}\text { Ciclista traumatizado } \\
\text { em AT* }\end{array}$ & 1 & 1,05 & 6 & 5,71 & 4 & 3,39 & 5 & 4,67 & 5 & 3,08 \\
\hline $\begin{array}{l}\text { Ocupante caminhonete } \\
\text { traumatizado em de } \mathrm{AT}^{*}\end{array}$ & 4 & 4,21 & - & - & 3 & 2,54 & 1 & 0,94 & - & - \\
\hline $\begin{array}{l}\text { Ocupante veículo trans- } \\
\text { porte pesado traumati- } \\
\text { zado em AT* }\end{array}$ & - & - & 1 & 0,95 & 1 & 0,85 & - & - & - & - \\
\hline $\begin{array}{l}\text { Ocupante ônibus trau- } \\
\text { matizado em AT* }\end{array}$ & - & - & - & - & - & - & 1 & 0,94 & - & - \\
\hline Total & 95 & 100 & 105 & 100 & 118 & 100 & 107 & 100 & 162 & 100 \\
\hline
\end{tabular}

Fonte: Sistema de Informações sobre Mortalidade/ Ministério da Saúde. AT- Acidente de Transporte 


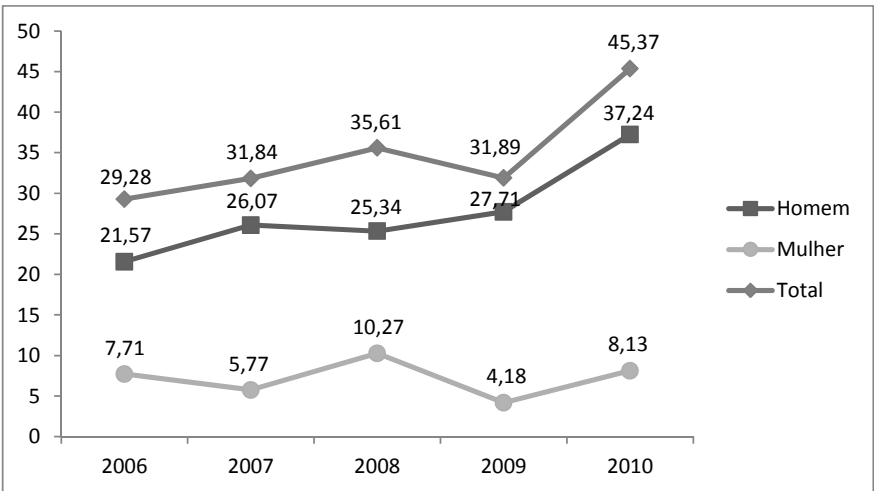

Figura 1-Coeficiente demortalidade por Acidente de Trânsito Terrestre, deacordocomo sexoentre2006e2010. Maringá-PR-Brasil,2010

Ao considerar os dados da Figura 1 e a variação percentual dos coeficientes de mortalidade por ATT do primeiro período (2006) e do último (2010), verificou-se aumento de $35,47 \%$ para a população analisada. E, ao se restringir ao sexo masculino esse valor elevou-se para 41,95\%; enquanto no sexo feminino houve variação percentual de apenas de 5,39\%. Ao realizar a diferença dos coeficientes de mortalidade por ATT em Maringá, nas duas coortes: 2006/2007 e 2009/2010 verificou-se que houve um aumento de $26,4 \%$ no coeficiente de mortalidade por ATT, como demonstrado na Tabela 2.

Por meio de relacionamentos de dados do total de óbitos geral em Maringá e óbitos por ATT, calculou-se o coeficiente de mortalidade para as variáveis: sexo e faixa etária, em ambas as coortes estudadas (2006/2007 e 2009/2010). Assim, a Tabela 3 apresenta o coeficiente médio e o odds ratio para óbitos por ATT da coorte 20062007, de acordo com as variáveis sociodemográficas.

Na Tabela 4, também se encontra o coeficiente médio e o odds ratio para óbitos por ATT, de acordo com as variáveis sociodemográficas, relacionadas à coorte 2009-2010. Conforme demonstrado na Tabela 4, do total de óbitos no município estudado, 226 foram por ATT. Todas as variáveis foram estatisticamente significativas.

Tabela 2 - Coeficiente de mortalidade por Acidentes de Trânsito Terrestre, segundo ano de ocorrência. Maringá, 2010

\begin{tabular}{lcccc}
\hline Período & $\begin{array}{c}\text { População } \\
(\mathbf{n})\end{array}$ & $\begin{array}{c}\text { Média das } \\
\text { populações }\end{array}$ & $\begin{array}{c}\text { Coeficiente Médio } \\
\left(\mathbf{x 1 0}^{-5}\right)\end{array}$ & $\begin{array}{c}\text { Diferença dos coeficientes } \\
(\mathbf{\%})\end{array}$ \\
\hline $2006-2007$ & 200 & 100 & 30,56 & $+26,4$ \\
$2009-2010$ & 269 & 134,5 & 38,63 & \\
\hline
\end{tabular}

Fonte: Sistema de Informações sobre Mortalidade/ Ministério da Saúde.

Tabela 3- Sexo e faixa etária, segundo óbito por Acidentes de Trânsito Terrestre. Maringá-PR-Brasil, 2006-2007

\begin{tabular}{lcccccc}
\hline Variáveis & $\begin{array}{c}\text { Total deóbitos Óbitos por } \\
(\mathbf{n})\end{array}$ & $\begin{array}{c}\text { \%TT* } \\
\text { (n) }\end{array}$ & $\begin{array}{c}\text { Coeficiente } \\
\text { Médio }\end{array}$ & p-valor & Odds ratio \\
\hline Sexo & 2889 & 156 & 78 & 49,87 & 0,000 & 3 \\
Masculino & 2352 & 44 & 22 & 12,91 & - & - \\
Feminino & & & & & & \\
Faixa etária & 369 & 76 & 13,26 & 9,69 & 0,000 & 3,9 \\
0 a 19 anos & 367 & 59 & 40,3 & 35,12 & - & - \\
20 a 39 anos & 1099 & 50 & 25,51 & 37,62 & 0,000 & 5,8 \\
40 a 59 anos & 3425 & 41 & 20,91 & 59,45 & 0,000 & 22 \\
60 e mais & $\mathbf{5 2 4 1 * *}$ & $\mathbf{1 9 6}$ & $\mathbf{1 0 0}$ & $\mathbf{6 2 , 7 8}$ & - & - \\
\hline Total & & & &
\end{tabular}

Fonte: Sistema de Informações sobre Mortalidade/ Ministério da Saúde.

*Acidentes por Trânsito Terrestre

**No total de óbitos constam três ignorados no ano de 2006. 
Tabela 4 - Sexo e faixa etária, segundo óbito por Acidentes por Trânsito Terrestre. Maringá-PR-Brasil, 2009-2010

\begin{tabular}{lcccccc}
\hline Variáveis & $\begin{array}{c}\text { Total de óbitos Óbitos por } \\
\text { (n) }\end{array}$ & $\begin{array}{c}\text { \%TT** } \\
\text { An) }\end{array}$ & $\begin{array}{c}\text { Coeficiente } \\
\text { Médio }\end{array}$ & p-valor & Odds ratio \\
\hline Sexo & & 226 & 84,01 & 67,92 & $* 0,000$ & 4,2 \\
Masculino & 3292 & 43 & 15,99 & 11,79 & - & - \\
Feminino & 2474 & & & & & \\
Faixa etária & & 31 & 11,52 & 13,75 & $* 0,000$ & 2,4 \\
0 a 19 anos & 284 & 106 & 39,4 & 43,58 & - & - \\
20 a 39 anos & 465 & 71 & 26,39 & 38,48 & $* 0,000$ & 4,6 \\
40 a 59 anos & 1189 & 61 & 22,69 & 86,68 & $* 0,000$ & 18,2 \\
60 e mais & 3824 & $\mathbf{2 6 9}$ & $\mathbf{1 0 0}$ & $\mathbf{3 9 , 8 5}$ & - & - \\
\hline Total & $\mathbf{5 7 6 6}$ & & & &
\end{tabular}

Fonte: Sistema de Informações sobre Mortalidade/ Ministério da Saúde. *Diferença estatística significativa. ** Acidentes por Trânsito Terrestre

\section{DISCUSSÃO}

Atualmente, os ATT constituem a primeira causa de morte por causas externas no Município de Maringá e ocupa o segundo lugar no Brasil, com números inferiores somente às agressões ${ }^{(4)}$. Contudo, a dimensão do problema relacionado aos ATT não se limita à alta mortalidade, em razão de que dentre suas diversas consequências, destacam-se as inúmeras sequelas e sofrimento nas famílias e na sociedade, bem como na elevação dos custos sociais e econômicos com alto impacto aos cofres públicos.

Ao exemplo da afirmativa anterior, pesquisa cujo objetivo foi descrever as estimativas de ocorrência de lesões decorrentes do trânsito e os fatores relacionados à população brasileira identificou que o ATT determinou o afastamento das atividades habituais em $30,7 \%$ dos acometidos; concluindo-se que a magnitude das lesões no trânsito justifica avançar em medidas preventivas e legislativas para a redução dos eventos ${ }^{(9)}$. Ademais, os sobreviventes de ATT, em especial adultos jovens e ocupantes de automóveis, quando sobrevivem, podem ser acometidos por incapacidade total ou funcional ${ }^{(10)}$.

Como se observa na Tabela 1, as causas de morte por ATT codificada pela CID-10 que obtiveram maior frequência foram, respectivamente: pedestre traumatizado em um acidente de transporte e motociclista traumatizado em um acidente de transporte.

$\mathrm{O}$ fato de pedestres e motociclistas serem vítimas predominantes de ATT coincide com outro estudo ${ }^{(11)}$. Em uma análise da situação de saúde e da agenda nacional e internacional de prioridades em saúde realizada pelo Ministério da Saúde apontou que ao se referir aos óbitos do ano de 2008, houve maior proporção por ATT nos grupos mais vulneráveis do sistema viário, sendo, respectivamente: pedestres $(24,2 \%)$; motociclistas $(23,4 \%)$; seguidos por ocupantes de veículos $(22,4 \%)^{(12)}$. Infere-se que, a mortalidade mais elevada no grupo de pedestre traumatizado em um acidente de transporte se deve à maior exposição corporal, com ausência de dispositivos de proteção para minimizar os danos decorrentes do impacto no momento do acidente.

A literatura aponta que nos atropelamentos predominam elevadas taxas de mortalidade em idosos ${ }^{(13)}$. Afinal, pessoas com 60 anos ou mais possuem menor agilidade para a travessia de ruas, e maior fragilidade diante às injúrias e danos corporais.

No que se refere aos motociclistas, é importante ressaltar que a elevada mortalidade nesse grupo pode ser decorrente do aumento da frota de motocicletas no país. E também pelo fato de que o único dispositivo de segurança passível de uso é o capacete, mantendo todo o restante do corpo desprotegido aos impactos. No Brasil, há crescente utilização da motocicleta no mercado de trabalho que, associada à dificuldade de sua visualização por outros motoristas, comportamento inadequados no trânsito e inobservância das leis, tem favorecido a ocorrência de $\mathrm{ATT}^{(11)}$.

Cumpre ressaltar que o ATT na categoria Ocupante de ônibus traumatizado em um acidente de transporte teve apenas uma ocorrência, datada em 2009 (Tabela 1). O número reduzido de óbitos na referida categoria pode estar relacionado ao fato de que os veículos de transporte coletivo apresentam maior proteção aos ocupantes, tanto pelo tamanho do veículo como pela baixa velocidade nas vias urbanas, esta causada pelo alto volume de tráfego; limite de velocidade reduzido e condições das vias públicas precárias ${ }^{(14)}$.

Ainda, com referência à Tabela 1 , se percebe que houve aparente redução da mortalidade por ATT em 
Maringá em 2009. O coeficiente de mortalidade, entretanto, não seguiu a mesma tendência do número de ocorrências de óbitos por ATT. Como se vê na Figura 1, o maior coeficiente foi alcançado no ano de 2008 e, embora em 2009 tenha havido uma redução, em 2010 foi o maior coeficiente de mortalidade dentre os períodos analisados. Em contrapartida, num estudo acerca da análise da mortalidade por ATT no Brasil, antes e após a referida Lei, evidenciou-se redução proporcional significativa no risco de morte nas capitais do país ${ }^{(15)}$.

As variáveis sexo e faixa etária mostraram forte associação com a ocorrência de óbito por ATT, tanto para a primeira (Tabela 3) como para a segunda coorte (Tabela 4). Ao comparar as coortes, nota-se que houve aumento do odds ratio para o sexo masculino de 3 para 4,2; com diminuição da razão de chances para óbito por ATT em todas as idades, quando comparada à categoria de referência, faixa etária 20 a 39 anos.

O perfil constatado nesta pesquisa está em consonância ao de outros estudos, que também revelam indivíduos do sexo masculino como vítima mais frequente, ou sob maior risco de sofrer $\mathrm{ATT}^{(13,16-18)}$. Isso porque, os homens têm comportamentos mais agressivos no trânsito do que as mulheres, muitas vezes manifestados por execução de manobras arriscadas, uso de bebida alcoólica e desrespeito às normas vigentes, além do fato de a profissão de condutor/motorista ser eminentemente masculina ${ }^{(18)}$.

Ao considerar que em ambos os períodos a variável faixa etária se associou ao óbito por ATT, à medida que a idade aumentava, evidenciou-se aumento do coeficiente de mortalidade e odds ratio (Tabelas 3 e 4). Infere-se que esses dados se relacionam ao fato e os adultos jovens possuírem menor experiência no trânsito; adotarem comportamentos tipicamente relacionados à imaturidade $\mathrm{e}$; recente obtenção da licença para dirigir ${ }^{(18)}$.

Para a redução dos ATT com os jovens, é considerado relevante o uso de estratégias peculiares que alcancem este grupo, para prevenir ou reduzir tais eventos, como: elaboração de estratégias de educação que visem à mudança nos hábitos e comportamentos, principalmente dos motoristas jovens; intensificação nas fiscalizações, com ações articuladas e integradas com os setores governamentais e não governamentais; e fornecimento de informações, por meio de educação continuada e sistematizada.

A maior proporção de óbitos por ATT no sexo masculino e na faixa etária entre 20 e 39 anos encontrada é corroborada por outro estudo ${ }^{(1)}$. No entanto, os coeficientes de mortalidade divergem do contexto nacional, pois as estatísticas de mortalidade recentes demonstram que nos países desenvolvidos a taxa de mortalidade é de apenas 10,3 óbitos por $100 \mathrm{mil}$ habitantes e nos países de média e baixa renda os coeficientes são 19,5 e 21,5 óbitos por 100mil habitantes, respectivamente. Para o Brasil, a taxa de mortalidade por esta causa é de 18,3 óbitos por 100 mil habitantes ${ }^{(4)}$.

Em contrapartida, a média foi de 34,98 óbitos por 100 mil habitantes no período de 2006 a 2010. Portanto, muito superior à taxa nacional. Mediante aos dados obtidos nesta pesquisa e aos resultados encontrados em outro estudo ${ }^{(19)}$, é preciso que haja maior investimento do Estado no desenvolvimento e manutenção de estratégias educativas, associadas às políticas públicas efetivas, no sentido de se reduzir os índices elevados de ATT.

Revela-se, portanto, como desafio ao país, gestores, educadores e à sociedade em geral, investir em mudanças de hábitos e comportamentos da população, no sentido de tornar as pessoas cautelosas e cumpridoras das leis de trânsito, associados à implantação de estratégias de promoção de ambientes seguros, à redução dos efeitos ocasionados pela relação beber e dirigir.

Com base nos resultados encontrados, considerase que o Plano Nacional de Redução de Acidentes e Segurança Viária precisa ser efetivado, pois o mesmo inclui articulação de estratégias, por meio de ações, metas e cronogramas de execução que visam à redução de acidentes e morte no trânsito. Para tanto, se faz necessário o envolvimento de todos os segmentos da sociedade.

\section{CONCLUSÃo}

Com base nos resultados apresentados, constatou-se que o coeficiente de mortalidade do município foi de $34,98 \%$, superior ao nacional, principalmente entre pedestres, motociclistas e homens na faixa etária de 20 a 39 anos. E na comparação das diferenças do coeficiente de mortalidade das coortes, houve um aumento de $26,4 \%$. Sugere-se, portanto, que haja formulação de estratégias consistentes de segurança rodoviária, apoiados por políticas, planos e programas nacionais que estabeleçam intervenções específicas, como melhoramento da infraestrutura viária; encorajamento e facilitação do uso alternativo de transporte; infraestrutura adequada das instituições de saúde ao atendimento das vítimas de trauma; além de práticas educativas para a população, em todos os segmentos 
da sociedade, com vistas ao exercício da cidadania e redução dos ATT.

Como limitações deste estudo, considera-se a ausência de dados atualizados acerca da mortalidade por ATT no Sistema de Informação sobre Mortalidade (DATA-SUS), o que dificulta a investigação mais recente sobre a temática e o curto período abordado. Deste modo, se faz necessária a continuidade de estudos que analisem períodos mais longos acerca da mortalidade por ATT e, que possibilitem revelar o impacto mais expressivo da medida restritiva do uso de álcool e da direção no trânsito.

\section{REFERÊNCIAS}

1. Ministério da Saúde (BR). Departamento de Análise de Situação em Saúde. Mortalidade por acidentes de transporte terrestre no Brasil. Brasília: Ministério da Saúde; 2007.

2. Malta DC, Filho SEM, Montenegro MMS, Mascarenhas MDM, Silva MMA, Lima CM et al. Atualização da lista de causas de mortes evitáveis por intervenções do Sistema Único de Saúde do Brasil. Epidemiol. Serv. Saúde. [Internet] 2011;20(3) [acesso em 20 set 2012]. Disponível: http://goo.gl/N2IQbN.

3. Ministério da Saúde (BR). Departamento de Análise de Situação em Saúde. Saúde Brasil 2010: Uma análise da situação de saúde e de evidências selecionadas de impacto de ações de vigilância em saúde. Brasília: Ministério da Saúde; 2011.

4. Brasil. Ministério da Saúde (BR). Departamento de Informática do SUS. Informações de saúde: Estatísticas vitais. Brasília (DF); 2012 [acesso em 15 jul 2012]. Disponível: http://www2.datasus.gov.br/DATASUS/ index.php?area $=0205$.

5. CID-10 - Classificação Estatística Internacional de Doenças e Problemas Relacionados à Saúde, Centro de Colaborador da OMS para Classificação de Doenças em Português- CBCD. 2008 [acesso em: 15 jul 2012]. Disponível: www.datasus.gov.br/cid10/V2008/cid10.htm.

6. Ministério da Saúde (BR). Plano nacional de redução de acidente e segurança viária para a década 20112020. Proposta preliminar. Brasília (DF); [Internet] 2008 [acesso em 20 ago 2012].Disponível:http://portal. saude.gov.br/portal/arquivos/pdf/plano_nac_reducao_ acidentes_comite_proposta_6611.pdf.

7. Matos KF, Martins CBG. Perfil epidemiológico da mortalidade por causas externas em crianças, adolescentes e jovens na capital do Estado de Mato Grosso, Brasil, 2009. Epidemiol. Serv. Saúde. [Internet] 2012;21(1) [acesso em 10 nov 2012]. Disponível: http:// goo.gl/jsr8CB. DOI: 10.5123/S1679-49742012000100005.

8. Brasil. Lei $n^{\circ}$. 11.705, de 19 de junho de 2008. Dispõe sobre o consumo de bebida alcoólica por condutor de veículo automotor. Diário Oficial da União, Brasília, 20 jun. 2008. Seção 1.

9. Malta DC, Mascarenhas MMDM, Bernal RTI, Silva MMA, Pereira CA, Minayo MCS et al. Análise das ocorrências das lesões no trânsito e fatores relacionados segundo resultados da PNAD Brasil, 2008. Ciênc. saúde colet. [Internet] 2011;16(9) [acesso em 20 ago 2012]. Disponível: http://dx.doi.org/10.1590/S141381232011001000005

10. Brito JMPX. Incapacidade por traumatismo raquimedular secundário a acidentes de trânsito. Coluna/Columna. [Internet] 2011;10(3) [acesso em 24 out 2012]. Disponível: http://dx.doi.org/10.1590/S1808-18512011000300001

11. Montenegro MMS, Duarte EC, Prado RR, Nascimento AF. Mortalidade de motociclistas em acidentes de transporte no Distrito Federal, 1996 a 2007. Rev. Saúde Públ. [Internet] 2011;45(3) [acesso em 20 ago 2012]. Disponível: http://goo.gl/IwQs4U

12. Ministério da Saúde (BR). Saúde Brasil 2009: uma análise de situação de saúde e da agenda nacional e internacional de prioridades em saúde. Brasília: Ministério da Saúde; 2010. 13. Léon-Marín L, Belon AP, Barros MBA, Almeida SDM, Restitutti MC. Tendência dos acidentes de trânsito em Campinas, São Paulo, Brasil: importância crescente dos motociclistas. Cad. Saúde Pública. [Internet] 2012;28(1) [acesso em 24 ago 2012]. Disponível: http://goo.gl/Z5SFpt

14. Bastos YG, Andrade, SM, Soares DA. Características dos acidentes de trânsito e das vítimas atendidas em serviço pré-hospitalar em cidade do Sul do Brasil, 1997/2000. Cad. Saúde Pública. [Internet] 2005;21(3) [acesso em 20 ago 2012]. Disponível: http://dx.doi.org/10.1590/S0102$311 \times 2005000300015$

15. Malta DC, Filho SEM, Montenegro MMS, Mascarenhas MDM, Silva MMA, Lima CM et al. Atualização da lista de causas de mortes evitáveis por intervenções do Sistema Único de Saúde do Brasil. Epidemiol. Serv. Saúde. [Internet] 2010;19(2) [acesso em 20 ago 2012]. Disponível: http://dx.doi.org/10.5123/S167949742010000200010

16. Martins CBG, Andrade SM, Soares DA. Morbidade e 
mortalidade por acidente de transporte terrestre entre menores de 15 anos no município de Londrina, Paraná. Cienc. cuid saude. [Internet] 2007;6(4) [acesso em 30 ago 2012]. Disponível: http://periodicos.uem.br/ojs/index.php/ CiencCuidSaude/article/viewFile/3880/2688

17. Camargo FC, Iwamoto HH. Vítimas fatais e anos de vida perdidos por acidentes de trânsito em MG, Brasil. Esc. Anna Nery. [Internet] 2012;16(1) [acesso em 30 ago 2012]. Disponível: http://goo.gl/b7zV01

18. Magalhães AF, Lopes CM, Koifman RJ, Muniz PT. Prevalência de acidentes de trânsito auto-referidos em Rio Branco, Acre. Rev. Saúde Públ. [Internet] 2011;45(4) [acesso em 10 nov 2012]. Disponível: http://goo.gl/ FPrxAw

19. Di Credo PF, Felix JVC. Perfil dos pacientes atendidos em um hospital de referência ao Trauma em Curitiba: implicações para a enfermagem. Cogitare enferm. [Internet] 2012;17(1) [acesso em 20 set 2012]. Disponível: http://ojs.c3sl.ufpr.br/ojs2/index.php/cogitare/article/ viewFile/26385/17578 